\title{
Recognition memory for automobiles: A developmental study
}

\author{
GRAHAM DAVIES and NOELLE ROBERTSON \\ University of Leicester, Leicester, England
}

\begin{abstract}
The ability of adults and children to recognize and name automobiles was compared with their competence at face recognition. Children, aged 7,9 , and 11 years, and adults $(N=198)$ observed pictures of 16 automobiles before trying to recognize them among 16 alternatives. One week later, they performed the same task with face stimuli. Performance improved with age on both tasks and was significantly better on faces for the adult and 11-year-old groups. Males were significantly superior to females at recognizing automobiles, whereas the trend was reversed for faces. Males were also more likely to be able to name automobiles correctly and to recognize them in an altered view. The results are interpreted in terms of knowledge and interest factors, enhanced recognition being mediated via the memory mechanisms of labeling and elaborated representation. The practical forensic implications and limitations of the current study are also discussed.
\end{abstract}

In 1989, there were some 140 attempted abductions of children by drivers from the streets and housing estates of the United Kingdom, an increase of $40 \%$ in 5 years (Abrams, 1990). Yet, little is known about children's competence to recognize or to accurately report details of automobiles. There are isolated case reports, such as that of the 10-year-old whose memory for internal automobile details led directly to the arrest of her abductor (Davies, Stevenson-Robb, \& Flin, 1988), but no normative data. The current study represents an initial investigation designed to assess the ability of children of different ages and sexes to recognize and name different makes of automobiles.

As befits an initial investigation, the method was a straightforward adaptation of the traditional study-test laboratory procedure. Subjects were shown photographs of different models of cars and then attempted to recognize those originals from a range of alternatives. This technique provides a rapid global assessment of recognition competence against which to set later studies that utilize less well controlled, though more ecologically oriented, procedures such as staged incidents. The large range of performance possible on such tasks also has the advantage of allowing contrasts between children and adult controls, as in the current study. One further advantage of such a study-test procedure is that it allows some comparison of recognition accuracy rates with another class of stimuli, about the developmental path of which much more is known: the human face.

The authors would like to thank Raph Gillett, Bill Williamson, David Mann, and John Ashworth for assistance in computing and statistics, and the staff and students of Swallowdale School, Melton Mowbray, and Whitehills School, Northampton. N. Robertson is in the Department of General Practice at the University of Leicester. Correspondence should be addressed to G. Davies, Department of Psychology, University of Leicester, University Road, Leicester LE1 7RH, England.
As Flin and Dziurawiec (1989) noted in their review, face recognition using the study-test technique shows a steady progression in competence from age 6 years to approximately 11 years. Flin and Dziurawiec could find little evidence for any strong influence of sex of observer on overall accuracy, although one major study (Cross, Cross, \& Daly, 1971) did find that girls were superior to boys when adult female faces formed the study set.

Predictions as to the performance of children at recognizing cars must, perforce, rely on a mixture of extrapolation and intuition. Studies of children's recognition ability with other relatively homogeneous stimulus sets such as flags and houses show improved performance from 7 to 15 years, with a regression at the onset of puberty (Flin, 1985), and a similar pattern might be predicted for automobiles.

The potential impact of sex of observer is more problematic. Knowledge and interest have a powerful impact on the levels of performance of children in memory tasks (Chi \& Ceci, 1986). Moreover, stereotypical differences in interests between the sexes have been shown to have a reliable effect upon amount and accuracy of recall in an eyewitness task (Powers, Andriks, \& Loftus, 1979). If boys are typically more interested in cars than are girls, then one might predict that the average recognition performance of boys might exceed that of girls. The role of appropriate verbal labeling of make or model might also play a role, given the pervasive influence of labeling upon recall and recognition in children (Davies, 1969). As regards faces, the use of adult female faces in the manner of Cross et al. (1971) might produce a reversal, with girls outperforming boys.

\section{METHOD}

\author{
Subjects \\ A total of 162 children drawn from two provincial primary schools \\ in the East Midlands participated in this study. Some 55 (28 male and
}


27 female) had a mean age of 7 years, a further 53 (24 male and 29 female) had a mean age of 9 years, and 54 ( 26 male and 28 female) had a mean age of 11 years. In addition, a group of 36 young adults (18 male and 18 female) drawn from the undergraduate population of the University of Leicester served as controls.

\section{Design}

A $2 \times 4 \times 2$ mixed design was employed in which sex and age served as between-subject factors and stimulus type (cars/faces) as a withinsubject factor. For the car test, each subject saw 16 cars before attempting to recognize them among 32 alternatives. For half the cars, orientation was maintained between study and test, whereas for the remainder, it was changed, the subset of targets that changed being counterbalanced within each age group. Exactly the same design was followed for the faces test.

\section{Stimuli}

All stimuli were specially prepared for this study. For cars, statistics were obtained for the top 50 best selling models in the United Kingdom in 1990. Monochrome photographs of 32 of these vehicles were then taken, and two views were obtained from a constant distance: a front view showing hood and windshield detail only, and a three-quarters view that gave more information on the proportions and profile of the vehicle. These photographs were then reproduced as slides, and 16 vehicles were selected as targets and 16 as distractors. To ensure recognition was by make and model of vehicle, license plate details were removed during photographic processing. Target and distractor sets were balanced by reference to market share and vehicle configuration (i.e., a sports coupe in the target set was balanced by allocating a sports coupe to the distractors, etc.).

For the faces, 32 female students, aged 17-25 years, who were unknown to any of the participants, were photographed both full face and in three-quarters pose. All pictures were monochrome and showed a head-and-shoulders view. Half the faces were randomly assigned to the target set, and the remainder formed the distractors.

\section{Procedure}

The subjects in each age range were split into two subsets to facilitate the counterbalanced conditions, and each subset was run separately as a group. The testing of the children was conducted in their classrooms, and the adult controls were tested in a university laboratory. All subjects were tested on cars first and were tested 1 week later on faces. For the cars, the subjects were warned that they would see some cars from the front and others from the side and that they would be required to recognize them at test irrespective of orientation. Orientation change was illustrated by three sample slides not included in the test set.

For the test, the 16 targets were shown sequentially for $10 \mathrm{sec}$ each with a 2 -sec interslide interval. There was a 5-min interval between study and test, during which a distractor task was presented. The test stimuli were then shown again, this time randomly interspersed with the distractor vehicles, with the same exposure intervals. The subjects were instructed to mark on an answer sheet the cars they thought they had seen before, irrespective of any change in orientation. When the answer sheets had been collected, the target set was shown again, this time with an instruction to the subjects to write down the make and model, if known, on a second answer sheet.

One week later, the same procedure was repeated, this time for the face-recognition task. The same counterbalanced groups and basic procedure and timing were used, except that the targets were not shown for a third time to elicit labels.

\section{RESULTS}

Each subject's answer sheet was scored for stimuli recognized, both correct (hits) and incorrect (false alarms). These were summed separately for both cars and faces, and two measures of performance, $d^{\prime}$ and $\beta$, were computed. These measures are derived from signal detection theory and give a measure of overall accuracy and response bias, respectively (Banks, 1970). In addition, to assess the impact of labeling, each subject's car answer sheet was further scored for ability to correctly label by make and/or model.

\section{Accuracy of Car Recognition Relative to Face Recognition}

Mean accuracy, as assessed by $d^{\prime}$ scores, is plotted as a function of age, sex, and stimulus type in Figure 1.

These data were subjected to a 2 (face/car) $\times 4$ (age) $\times 2$ (sex) multivariate analysis of variance (MANOVA), which revealed significant main effects for age and stimulus type. There was a general improvement in overall performance with age $[F(3,190)=71.61, p<.001]$, and faces were generally better recognized than cars $[F(1,190)=26.08, p<.001]$. The latter result was qualified by a significant age $\times$ stimulus interaction $[F(3,190)=10.55, p<.001]$. The simple main effects of this interaction indicated that although there was no significant difference between the two classes of stimuli
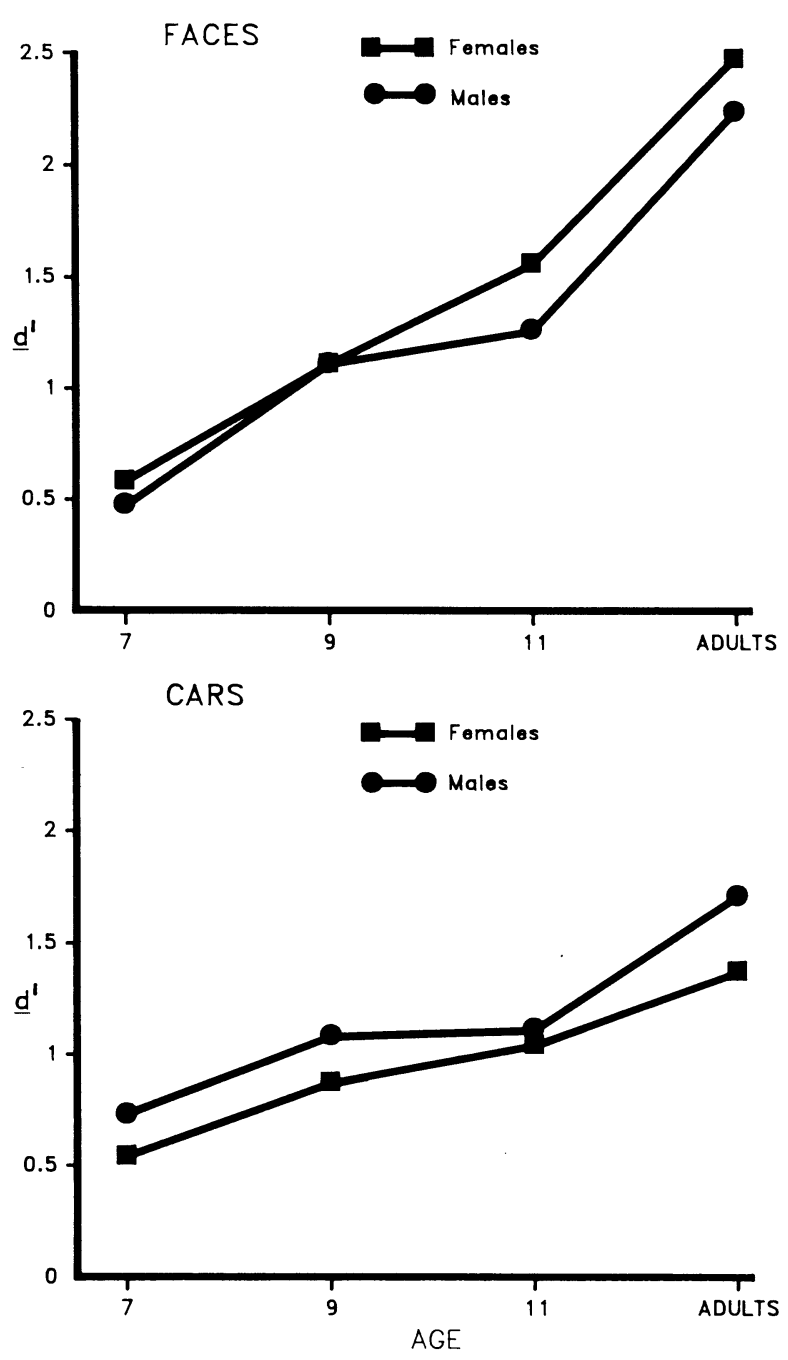

Figure 1. Mean accuracy $\left(d^{\prime}\right)$ for car and face recognition as a function of age and sex. 
at age 7 , faces were consistently better recognized than cars by all the older age groups $(p<.001$ for adults and 11-year-olds).

The sex of the subject had no overall effect $(F<1)$ but did significantly interact with stimulus type $[F(1,190)=9.89, p<.01]$. Exploration of the simple main effects of this interaction confirmed the predicted pattern: although there was a trend for females to outperform males on face recognition $(p<.10)$, males were superior to females in the recognition of cars $(p<.05)$. All other interactions proved insignificant $(F<1)$.

To assess the degree to which proficiency at recognizing faces was related to proficiency at recognizing cars, the relationship between the two was examined using a series of Pearson product-moment correlations. Performance on the two tasks was essentially unrelated at the individual age levels, although there was a significant positive correlation when the data were collapsed across ages for both males $(r=.35, p<.001)$ and females $(r=.46$, $p<.001)$

Response bias, as assessed by $\beta$ scores, was investigated using the same form of analysis as for $d^{\prime}$. Response bias was again significantly influenced by age $[F(3,190)=$ $19.92, p<.001]$ and stimulus type $[F(1,190)=125.06$, $p<.001]$, and this was qualified by an age $\times$ stimulus interaction $[F(3,190)=21.17, p<.001]$. However, exploration of the simple main effects of this interaction revealed a very different pattern from the accuracy data. The subjects adopted a consistently more lax criterion toward cars than faces at all ages (all $p s<.01$ or better), but the pattern of performance with age differed for cars and faces. Simple main effects analysis showed that for cars, response bias did not fluctuate significantly with age, but for faces, the adults were significantly more conservative in their decision making than were any of the child groups $(p<.001)$, which did not differ among themselves. In summary, the analysis suggests that there is less readiness among all participants to identify a face than a car as having been seen before, with the effect being particularly marked for adults.

\section{Effect of Orientation}

The impact of changed orientation was examined by comparing hit rates for changed versus maintained views at test. For the purposes of analysis, the results of the car and face tests were treated separately and subjected to MANOVAs that used age, sex, and orientation as main effects.

For cars, there was a significant main effect for orientation $[F(1,191)=28.00, p<.001]$ such that cars that were tested in the same view as at study $(M=6.32)$ were more likely to be correctly recognized than those that changed $(M=5.74)$. However, this main effect was qualified by a significant sex $\times$ orientation interaction $[F(1,191)=11.38, p<.001]$. Examination of the simple main effects of this analysis indicated that although males did not differ significantly in their hit rates on same and changed stimuli, females did significantly better $(p<$
.01) when orientation was maintained $(M=6.42)$ than when it changed $(M=5.51)$. The orientation effect was uninfluenced by the age of the subject $(F<1)$.

For faces, there was again a significant effect for orientation $[F(1,153)=4.61, p<.05]$ such that faces seen in the same view as at study $(M=4.94)$ were slightly better recognized than those that changed $(M=4.62)$. Once again, sex interacted with orientation $[F(1,153)=$ $13.86, p<.001]$. Simple main effects analysis confirmed that although females did not differ significantly in their hit rate on faces that maintained or altered in orientation between study and test, males did significantly better $(p<.01)$ when view was maintained $(M=5.38)$ than when it changed $(M=4.49)$. On this occasion, orientation also interacted significantly with age $[F(2,153)=10.95$, $p<.001]$. Examination of the simple main effects of this analysis indicated that this reflected the disproportionate contribution of the 9 -year-olds $(p<.01)$ to the overall effect of maintained orientation.

In summary, when cars are employed as stimuli, males generally are more able than females to cope with transformation at test, whereas the position is exactly reversed when female faces form the stimulus set. Thus, these data amplify and confirm the gender effects observed for recognition accuracy overall.

\section{Knowledge of Car Names}

The mean number of vehicles correctly named by make and/or model was tabulated and subjected to an ANOVA that used age and sex as main effects. This confirmed a significant improvement in knowledge from a mean of $7 \%$ at age 7 to $72 \%$ correct for the adults $[F(3,190)=83.44$, $p<.001]$. Once again, there was a significant superiority for males over females $[F(1,190)=22.93, p<$ $.001]$, for all groups except the 7-year-olds, who were responsible for a significant age $\times$ sex interaction $[F(3,190)=4.01, p<.01]$.

Tabulating the frequency of correct naming indicated wide variation in the frequency with which different cars within the test set were recognized. Agreement between adult and child observers on the relative ease of naming different cars was highly significant $[r(14)=.71, p<$ $.001]$. Examination of the relationship between frequency of naming and overall sales showed no simple relationship, suggesting that recognizability was not just a simple function of familiarity.

\section{Influence of Labeling on Recognition Accuracy}

A final series of analyses examined the impact of a subject's knowledge of a car's name on his/her ability to recognize it at test. The proportion of times each of the 16 cars was labeled correctly and also recognized was computed, together with the number of times the same car was not labeled but still recognized. Overall, labeled cars were also recognized on $82 \%$ of occasions, whereas unlabeled cars were recognized on $74 \%$ of occasions, confirming a small but significant impact of labeling upon recognition $[t(15)=2.55, p<.025$, one tailed]. 


\section{DISCUSSION}

It is evident that the recognition of automobiles, like the recognition of faces, shows a clear improvement with age, although judged by the relevant results, growth in competence is somewhat slower. Moreover, performance on the two tasks appears to be mediated by stereotypical interest factors in all but the youngest age group tested. Females enjoyed a marginal advantage over males in the recognition of female faces, consistent with the earlier finding of Cross et al. (1971). Males, on the other hand, showed a somewhat larger advantage over females in the recognition of cars.

Levels of performance on the two tasks were independent for subjects of the same age, suggesting that generalized discriminative competencies played little part in determining performance. However, caution is necessary in interpreting these insignificant correlations in the absence of a second test of face recognition, given the frequently low test-retest reliability of such stimuli (Woodhead \& Baddeley, 1981).

Although generalized recognition competencies appear to play little role in determining within-group variance, they almost certainly contribute to between-group differences, as reflected in the significant overall correlations between the two tasks when the data are collapsed across age. In addition, such age-related improvements may be driven by the greater social significance that both cars and female faces have for adults as opposed to young children. Support for this in relation to cars is provided by the large jump in labeling rates between the 11-year-old and adult groups (an increase from $36 \%$ to $72 \%$ ), probably reflecting the involvement of the latter group in car driving and ownership: all but 4 of the adult subjects possessed driver's licenses.

The interest factor seems to be the most plausible explanation of the gender differences observed in recognition of cars and faces. McKelvie (1981) suggests that any trend for females to exceed males on recognition of female faces may reflect stereotypical interests in cosmetics and facial appearance. How the interest factor mediates recognition accuracy for cars is not so clear. At all ages except 7 years, males more readily name cars correctly than do females. Consistent with the stimulus predifferentiation literature (Davies, 1969), such labeling facilitates subsequent recognition. However, the effect is modest and insufficient in itself to explain the consistent superiority of males over females. Only a minority of vehicles were labeled appropriately by subjects of either sex, and a substantial number of positive responses could not be attributed to labeling.

Another plausible explanation would be the greater knowledge of vehicles that the average male subject possessed relative to his female counterpart. As Ellis and Deregowski (1981) demonstrated, interest in and familiarity with a class of stimuli such as faces is likely to translate into more flexible and generalized representations of such stimuli in memory. In their study, black adults were more able to readily identify black faces that were transformed in pose from study to test than were whites, whereas whites showed a parallel superiority over blacks on white faces. It is evident that a similar pattern was present in the current study for faces and for cars; on the latter stimuli, males were more able to correctly identify vehicles in a changed orientation than were females, whereas there was little difference between the sexes when the stimuli had to be recognized in the same view. Once again, however, the effects, although highly significant, are modest in absolute terms.

What are the practical implications of these findings? Although caution is necessary before extrapolating too far from such a basic laboratory study to the real-world problems of child abduction, the current results appear to have some interesting implications. There is the con- sistent difference in criterion adopted by all subjects, and adults in particular, in their readiness to identify a stimulus as old when confronted with a face as opposed to a car. If a similar finding is confirmed by studies in field settings, caution may need to be exercised by the police in interpreting the selections made by naive witnesses from the collections of car photographs held by many constabularies.

Do the current data provide any guidance as to how the police might isolate witnesses who are likely to be more or less accurate in identifying vehicles? Clearly, to suggest that these results show that they should put more weight on the word of a male than a female witness would be entirely inappropriate. Although the current findings are of theoretical interest, the differences between the sexes, although significant, are modest and are not of practical utility. At all ages, there were female subjects whose scores exceeded those of males, and vice versa. Likewise, the superior average performance of the adult group masked considerable variation in the performance of the children, with some of the 9- and 11-year-olds producing recognition performance well within the adult norms. The best advice, based on the current study, would be to examine a witness's general competence at recognizing and particularly at labeling automobiles. The witnesses of either sex or any age who show interest in and prowess at such tasks are likely to be the most reliable and valuable observers of vehicle information.

\section{REFERENCES}

Aвrams, F. (1990, November 18). Peter Pan the failed persuader. Sunday Correspondent, p. 12.

BANKs, W. P. (1970). Signal detection theory and human memory. Psychological Bulletin, 74, 81-99.

CHI, M., \& CECI, S. J. (1986). Content knowledge and the reorganization of memory. In H. W. Reese (Ed.), Advances in child develop ment and behavior (Vol. 20, pp. 1-37). New York: Academic Press.

Cross, J. F., Cross, J., \& Daly, J. (1971). Sex, race, age, and beauty as factors in recognition of faces. Perception \& Psychophysics, 10 , 393-396.

DAviES, G. (1969). Recognition memory for pictured and named objects. Journal of Experimental Child Psychology, 7, 448-458.

Davies, G., Stevenson-Robb, Y., \& Flin, R. (1988). Tales out of school: Children's memory for an unexpected event. In M. M. Gruneberg, P. E. Morris, \& R. N. Sykes (Eds.), Practical aspects of memory (Vol. 1, pp. 122-127). Chichester: Wiley.

Ellis, H. D., \& Deregowski, J. B. (1981). Within-race and betweenrace recognition of transformed and untransformed faces. American Journal of Psychology, 94, 27-35.

FuIN, R. H. (1985). Development of visual memory: An early adolescent regression. Journal of Early Adolescence, 5, 259-266.

Fuin, R., \& DzIURAWIEC, S. (1989). Developmental factors in face processing. In A. W. Young \& H. D. Ellis (Eds.), Handbook of face processing (pp. 335-378). Amsterdam: North-Holland.

MCKelvie, S. J. (1981). Sex differences in memory for faces. Journal of Psychology, 107, 109-125.

Powers, P. A., ANDriks, J. L., \& LofTus, E. F. (1979). Eyewitness accounts of females and males. Journal of Applied Psychology, 64, 339-347.

WOODHEAD, M. M., \& BADDELEY, A. D. (1981). Individual differences and memory for faces, pictures, and words. Memory \& Cognition, 9, 368-370.

(Manuscript received September 14, 1992.) 